\title{
CULTURA DE INNOVACIÓN COMO ESTRATEGIA PARA EL FUTURO
}

\author{
Alisson Andrea Núñez Martínez \\ Universidad Santo Tomas
}

\section{Resumen}

La siguiente ponencia pretende exponer la importancia de la cultura de la innovación, partiendo de que actualmente la innovación no se limita únicamente a los avances tecnológicos o al lanzamiento de nuevos productos o servicios, ahora este concepto abarca una necesidad, y es la de generar una cultura innovadora en la que cada sujeto tenga la oportunidad de aportar social y económicamente a un país, desarrollando una capacidad sólida para innovar.

Para esto es importante romper los paradigmas de todas las partes interesadas en el crecimiento a través de la innovación, involucrando políticas que aumenten la inversión en gestión del conocimiento para que se promueva un entorno donde sea posible la transformación de ideas en resultados y es aquí donde convertir la cultura de la innovación en un objetivo social debe ser la labor en futuro cercano de todos los países, como en este caso Barcelona.

\section{Palabras clave}

Cultura, crecimiento, innovación, conocimiento, estrategia. 


\section{CULTURA DE INNOVACIÓN COMO ESTRATEGIA PARA EL FUTURO}

\section{INTRODUCCIÓN}

El entorno y sus cambios constantes hacen que la innovación sea la principal opción estratégica para el futuro.

En la actualidad cada sociedad debe afrontar los retos diarios que traen los cambios tecnológicos y avances científicos, obligando constantemente a estar preparados para estos acontecimientos y evaluando la capacidad de respuesta de cada actor social a la hora de asumir estrategias, para conseguir competitividad o simplemente supervivencia.

Pero a su vez estos cambios vienen acompañados de soluciones desarrolladas a lo largo del tiempo, que ayudan a obtener una permanecía económica y una estabilidad social. Es aquí donde la innovación es para muchos su principal aliado, optando por direccionar sus recursos a la creación de valor, fomentando la creatividad, el emprendimiento y el uso de la tecnología como herramienta para el desarrollo económico y social.

Según el profesor Rafael Vesga estos asuntos se deben realizar en tres niveles; el nivel macro del país, el nivel intermedio de las empresas y el nivel básico de los individuos (Vesga, 2006), donde es sumamente importante que se establezca una cultura innovadora en cada uno de estos niveles para logar un beneficio colectivo. Es por esto que tengo como propósito exponer esta importancia, tomando como referencia la experiencia en Barcelona, la visita realizada a la empresa Barcelona Activa y la lectura de documentos que abordan este tema y sirven como guía para exponerlo.

Posteriormente se concluirá con un análisis acerca de la cultura de la innovación y el emprendimiento en Colombia.

\section{ANTECEDENTES}

A continuación se realiza una breve conceptualización de los términos más importantes para la elaboración y por supuesto, para la comprensión de esta ponencia.

\subsection{Innovación}

El termino innovación procede del latín "innovare" que indica acción y efecto de innovar o en su sentido más amplio creación o modificación de un producto, y su introducción en un mercado. (Real Academia Española, 2015)

La cantidad de definiciones y enfoques de este concepto es bastante amplia por su adopción en diferentes áreas del conocimiento, por tal motivo para esta ponencia se tomo los aportes realizados por Joseph Schumpeter y Peter Drucker. 
Schumpeter fue el primer economista en identificar la innovación como el motor para el cambio económico ya que este cambio es el resultado de un desequilibrio dinámico producido por un empresario innovador. (Veiga, 2008)

Su pensamiento parte del concepto de "corriente circular" el cual describe la vida económica como un sistema en equilibrio que se consigue a través de alteraciones en variables exógenas; estas variables generan presiones en el aparato productivo que posteriormente se convierten en innovaciones en el sistema económico.

Un claro ejemplo se presenta cuando un empresario desarrolla un producto novedoso, obtiene grandes beneficios durante un tiempo, hasta que los competidores lo copian y se vuelve a un beneficio normal. Este proceso se repite constantemente, lo que hace que el empresario sea considerado el impulsor del proceso de avance tecnológico. (BBVA, 2012)

Dicho esto, el desarrollo económico surge de la producción de nuevos bienes y/o servicios o en la optimización de métodos para su realización.

Siguiendo el enfoque de Schumpeter, Peter Drucker entiende la innovación de dos maneras, como el cambio en el rendimiento de los recursos y la innovación como la acción de cambiar el valor y la satisfacción obtenida por el consumidor.

Afirma que el único propósito para una empresa es obtener un cliente, el cual exige dos funciones fundamentales: comercialización e innovación.

Para esta última función Drucker propone seis fuentes para identificar oportunidades de innovación, las cuatro primeras dentro de la organización, las cuales implican lo inesperado, lo incongruente, la necesidad de un proceso y el cambio en la estructura de la industria y del mercado, en el segundo conjunto se encuentran los cambios producidos fuera de la empresa y está constituido por cambios en la población, cambios en la percepción y nuevos conocimientos. (Soto Flores \& Medellín Cabrera, 2010)

Ninguna de las fuentes es más productiva que la otra, sin embargo las innovaciones basadas en nuevos conocimientos son consideradas por Drucker como la productora de oportunidades menos predecibles y confiables las cuales requieren más tiempo y presentan más desafíos para los empresarios.

De acuerdo al pensamiento de Drucker, la innovación es una actividad esencialmente económica, pero también empresarial y social, resultado de una disciplina sistemática, organizada y de aprovechamiento de oportunidades producidas de los cambios dentro y fuera de la organización.

Por esto la innovación obliga a los empresarios a poner en práctica actividades y estrategias específicas y diferentes para detectar oportunidades de innovación favorables.

\subsection{Cultura}

Al igual que el término innovación, la definición de cultura tiene muchos significados interrelacionados aportados y analizados por diferentes autores. 
Por consiguiente este texto se basa en las siguientes definiciones

- Para Alcina José (Alcina Franch, 1989) "la cultura es el conjunto de ideas, hábitos y actividades, de carácter técnico, económico, social, espiritual y lingüístico, creado por la sociedad que se transmite de generación en generación por medio de la tradición, al mismo tiempo que el producto de una tremenda fuerza innovadora"

- Según Spradley \&McCurdy (McCurdy \& Spradley, 1975)“la cultura es definida como el conocimiento adquirido que las personas utilizan para interpretar su experiencia y generar comportamiento"

\subsection{Barcelona}

Para cumplir con el objetivo de este trabajo considero conveniente realizar una breve descripción de Barcelona, la cual incluye algunos indicadores económicos que aportan una visión más amplia sobre la ciudad.

Barcelona es la capital de Cataluña, con más de 2.000 años de historia cuenta con 1.615.448 habitantes. Es el núcleo central de una región metropolitana de 4.777.042 habitantes y es quinta en Europa por su población. Su mercado directo es de más de 18 millones de habitantes y abastece desde Valencia hasta el sudeste de Francia, y desde las Islas Baleares hasta Aragón.

Es la única ciudad del Sur de Europa que concentra 5 kilómetros lineales, uno de los mayores puertos del Mediterráneo, una estación de tren de alta velocidad, un aeropuerto internacional, autopistas conectadas a la red europea, el segundo mayor recinto ferial de Europa y una de las mayores zonas logísticas del sur de Europa.

Barcelona es líder de una gran área económica, con una dilatada tradición industrial y un denso tejido empresarial. La ciudad apuesta por la economía y las actividades intensivas en conocimiento, especialmente los servicios avanzados y las nuevas actividades económicas.

El sector industrial todavía tiene un peso relativamente importante, con empresas competitivas, especializadas y orientadas hacia la exportación. Sin embargo, Barcelona se encuentra en un proceso de transición ordenada hacia la economía del conocimiento. Actualmente cuenta con $\mathbf{8 8 . 0 0 0}$ trabajadores en industrias creativas y es decima en el mundo por producción científica

El área de Barcelona es la sede de 438.385 empresas, el $14 \%$ del total de España, especialmente de pymes. La tasa de actividad emprendedora de la población residente (18-64 años) se sitúa en 6.5\% en 2012 superando las tasas de Francia Finlandia o Alemania, así como la media española $5.7 \%$. El $48 \%$ de la población activa de la ciudad trabaja en servicios intensivos en conocimiento.

Algunos sectores estratégicos en Barcelona son tecnologías de la información y la comunicación, biotecnología y ciencias de la vida, energía, diseño, movilidad sostenible y aeronáutica, educación superior e investigación, logística y transporte de mercancías, turismo, entre otras. (Barcelona activa, 2014) 


\subsection{Barcelona activa}

Es una organización anónima municipal, ejecutora de las políticas de promoción económica del Ayuntamiento de Barcelona.

Desde 1986 impulsa el m crecimiento económico de Barcelona y su ámbito de influencia apoyando a las empresas, la iniciativa emprendedora y el empleo, al tiempo que promociona la ciudad internacionalmente y sus sectores estratégicos, en clave de proximidad al territorio.

En esta organización acompañan a las personas emprendedoras desde que tienen una idea de negocio hasta que ponen en marcha su empresa, promoviendo la iniciativa emprendedora y el posicionamiento de Barcelona como referente de ciudad emprendedora.

El modelo mixto, sus herramientas digitales y metodologías para apoyar el emprendimiento y las empresas desarrolladas por Barcelona Activa han sido transferidas a entornos tales como Casablanca (Marruecos), Ciudad del Cabo (Suráfrica), Brasil, Santiago de Chile (Chile), Buenos Aires (Argentina), Montevideo (Uruguay), Bogotá y Medellín (Colombia), Quito (Ecuador), México y Roma (Italia) entre otros (Barcelona Activa).

Se organiza en dos direcciones ejecutivas de prestación de servicios y cinco direcciones corporativas de carácter transversal, para desarrollar las siguientes líneas de trabajo (Ayuntamiento de Barcelona. Barcelona Activa, 2014):

\subsubsection{Dirección Ejecutiva de Servicios a las Empresas y Empleo}

- Empresa: apoyar en la mejora de la competitividad de las empresas de la ciudad de Barcelona a través del fortalecimiento de relaciones con las empresas tractoras y promoviendo una oferta de servicios permanentes que fomenten la creación de riqueza y empleo en la ciudad

- Servicios: Oficina de Atención a la Empresa

- Programas: Axelera, Learning to grow, Sales management, Mentoring, Reinvierte en future, entre otros.

- Emprendimiento: promover la iniciativa emprendedora y acompañar a las personas emprendedoras en el proceso, desde la idea de negocio a la puesta en marcha de la empresa.

- Servicios: Difusión y orientación en emprendimiento, Asesoramiento y acompañamiento a la creación de empresas, Servicios en línea y Actividades formativas en creación de empresas

- Programas: Creación de empresas en sectores estratégicos, Creación de empresas en sectores tradicionales, Creación de empresas para colectivos y Programa "Raising stars.

- Capacitación profesional y empleo: orientar y capacitar a las personas a lo largo de su carrera profesional, de acuerdo con los requerimientos del mercado y las empresas. Favorecer el encuentro cualitativo entre demanda y 
oferta de trabajo, y dar respuesta a las necesidades de las empresas en términos de capital humano.

- Servicios: Asesoramiento personalizado, Orientación, búsqueda de empleo y desarrollo profesional, Orientación en los centros educativos, Web Barcelona Treball y Barcelona Crea Ocupación.

- Programas: Programa de Inserción Sociolaboral (PISL), Mujer impulso, Programa Personalizado para la Búsqueda de Trabajo (Proper, Trabajo en los barrios, Jóvenes por el empleo, entre otros.

- Formación: mejorar las competencias de los ciudadanos, profesionales y empresas, para hacer de Barcelona una ciudad más competitiva.

- Servicios:

- Formación complementaria a los servicios y programas de empresa, emprendimiento y empleo

- Formación en capacitación tecnológica. Cibernàrium

\subsubsection{Dirección Ejecutiva de Promoción}

> Promoción de la ciudad: potenciar los flujos, intercambios y relaciones económicas de Barcelona con el exterior para posicionar la marca Barcelona e impulsar la economía y el liderazgo empresariales de Barcelona y el Área Metropolitana.

Acciones y proyectos:

- Promoción de la ciudad en el exterior

- Alianzas económicas de ciudades

- In-city promotion

- Acompañamiento de proyectos

- Promoción de los sectores estratégicos: mejorar la competitividad de las empresas y de la ciudad, así como maximizar su liderazgo y posicionamiento, tanto a nivel local como internacional, en todos aquellos sectores considerados estratégicos, con especial incidencia en los sectores TICMobile, logística, agroalimentario, energía y movilidad sostenible, biotecnología, aeroespacial y educación superior.

- Promoción del turismo y eventos: impulsar y coordinar las acciones relacionadas con la gestión del impacto que supone el turismo en la ciudad a fin de que, manteniendo su importancia económica, mejore la calidad de vida de los ciudadanos. Promover la atracción, aterrizaje y fidelización en la ciudad de eventos de interés económico.

- Promoción del territorio y del comercio: desarrollar una promoción económica de proximidad trabajando con los distritos para identificar y promover nuevas potencialidades económicas e impulsar el rol del pequeño comercio y contribuir a su modernización. 


\subsubsection{Direcciones corporativas}

- Recursos Económicos: optimizar la gestión de las áreas de Economía y Finanzas, Servicios Generales, Captación de Recursos, Estudios y Mantenimiento, estableciendo las políticas y criterios necesarios para alcanzar los objetivos de la organización.

- Recursos Humanos: definir las políticas y estrategias en las materias que le son propias, como la selección de personas, la formación, las relaciones laborales y la prevención, para optimizar la gestión de los recursos humanos.

- Sistemas de Información y Organización: coordinar los proyectos de infraestructuras de telecomunicaciones que se puedan desarrollar con el fin de garantizar la seguridad y calidad de los sistemas de información de Barcelona Activa. Mejora de la organización mediante el diseño de procesos y sistemas que garanticen la calidad y eficiencia en la prestación de los servicios.

- Servicios Jurídicos: asesorar jurídicamente a todos los departamentos de Barcelona Activa de acuerdo con la normativa vigente, a fin de asegurar la legalidad de todas las actuaciones.

- Comunicación y Marketing: participar en la definición y ejecución de la política de comunicación y de marketing de

Barcelona Activa cuenta con una red de equipamientos avanzados para impulsar el conjunto de sus actuaciones de promoción económica, algunos de ellos son:

- Sede central, que alberga la dirección de la empresa, los servicios centrales de la Dirección de Gestión estrategia, y el equipo que impulsa las actuaciones en materia de promoción.

- Centro para la Iniciativa Emprendedora Glòries, el equipamiento de referencia en la ciudad para la creación de empresas.

- Incubadora de empresas Glòries, espacios y servicios de incubación para nuevas empresas innovadoras.

- Incubadora de empresas Almogàvers, un entorno de incubación para el crecimiento de nuevas empresas innovadoras de la ciudad, gestionado en colaboración público-privada.

- Parque Tecnológico Barcelona Nord, entorno de incubación de empresas innovadoras de base tecnológica en fase de crecimiento.

Estos equipamientos se complementan con una red de proximidad que incluye trece antenas de capacitación tecnológica inicial en bibliotecas públicas, nueve puntos de atención ocupacional distribuidos por el territorio de la ciudad, trece espacios de referencia para los jóvenes de la ciudad (puntos de información juvenil y espacios jóvenes) donde se ofrece el programa "Barcelona Trabajo jóvenes", y veintiséis equipamientos de la ciudad donde han recibido formación los comerciantes de la ciudad con el programa "Abierto al futuro".

\section{OBJETIVO}

Presentar la importancia de la cultura innovadora a partir de las prácticas, programas e iniciativas desarrollas en Barcelona Activa. 
Específicos:

- Establecer el aporte económico de Barcelona activa.

- Analizar la situación actual de Colombia frente a temas relacionados con la innovación.

\section{REVISIÓN DE LA LECTURA}

\subsection{Cultura de innovación}

Ing. Lorenzo G. Rodriguez Rios Delphi Automotive Systems Fundación Premio nacional de tecnología

En este artículo se presenta la importancia de ver la innovación como una ventaja competitiva sustentable que debe mirarse como un estilo de vida ya que muchos perciben la innovación como un método donde se aplica una serie de procedimientos sin considerar lo más importante: la creación de una cultura de innovación.

Según el autor este estilo de vida se logra a partir de un compromiso global a largo plazo, donde cada persona involucrada se convierte en agente de cambio con espacios ideales para el surgimiento de ideas, la experimentación de nuevas soluciones, impulsando la creatividad y el uso de la tecnología.

Este proceso puede tomar varios años pero los resultados que se obtienen son muy importantes para las organizaciones que lleguen a la cima de la cultura de innovación ya que no necesitan hacer evaluaciones comparativas de la competencia, estas organizaciones se vuelven el modelo que la competencia tiene que seguir.

\subsection{Innovación y cultura: la medida social de la innovación}

Armando Menéndez Viso Revista de filosofía moral y política Universidad de Oviedo

Este artículo pretender ofrecer pistas sobre cómo podría medirse, ya no la innovación como efecto, sino la innovación como acción, es decir, aquellas condiciones que posibilitan la innovación. Se argumenta que (Menéndez Viso, 2013) "las medidas deben basarse en ciertas variables culturales, que ponderen la diversidad de los individuos y grupos de una sociedad, como indicador de su capacidad combinatoria."

I. Para lograr entender aquellas medidas el autor plantea la innovación desde dos perspectivas conceptuales: la innovación como acción y la innovación como efecto.

Innovación como efecto: son los resultados de la acción de innovar cuando dicha acción tiene éxito, un claro ejemplo son los cuatros tipos de innovación 
mencionados en el Manual de Oslo (OCDE, 1997) ("de producto, de mercadotecnia, de proceso y de organización")

Innovación como acción: aquella que conduce a la producción de efectos innovadores, creando o mejorando significativamente un proceso, producto, método o práctica. Esta perspectiva de innovación cesa cuando ha producido un efecto exitoso a partir de una acción innovadora.

II. En general, la innovación como acción es el medio para la innovación como efecto y esta se entiende deseable porque aumenta la riqueza en ambos sentidos; a los oferentes porque les permite aumentar sus ventas, generar más utilidades, reducir costos, mejorar frente a la competencia, a los demandantes porque permite la mejor satisfacción de sus necesidades y el aumento de bienestar.

La cantidad y calidad de los bienes disponibles resultan de la capacidad productiva, donde el papel de la innovación consiste en impulsar dicha capacidad fomentando el uso de la tecnología para logar crecer a nivel de producción y actividad económica; presentándose como único instrumento para mejorar la calidad de vida. Por esto la innovación se convierte en un deseo generalizado.

Seguramente por todo esto, llamar a algo innovador es valorar de forma positiva y desde luego deseable, que amplia y supone progreso

A partir de esta afirmación el autor propone otro objetivo de la innovación, no desde la búsqueda de soluciones o satisfacción de necesidades sino del deseo de aumentar la capacidad de acción que posteriormente se verá traducida a mejoras en la calidad de vida.

III. La visión cultural de la innovación no se centra en el cómo y dónde se engendra el nuevo producto sino como y donde se implanta. Esta forma de ver la innovación traslado la fuente de valor de los objetos a los sujetos, es decir, el verdadero valor de las cosas lo otorga el sujeto, no por las cualidades del objeto, sino por las necesidades que cubre.

Este pensamiento que pone al sujeto como fuente principal de valor vuelve a la innovación como un patrón cultural ya que las innovaciones solo surgen en sociedades y entre sus efectos esta siempre el definirlas, es decir un producto efectivamente innovador es aquel que crea una comunidad entorno a él y se distingue de otras comunidades con innovaciones diferentes.

A partir de esto no es casualidad que la teoría económica haya incorporado lo cultural a lo económico y con ello, ofrecer una economía más dinámica donde la innovación es una fuerza motriz fundamental.

IV. Para el autor la innovación es un concepto con varias tensiones internas donde ambas visiones, la tecnología y cultural, coinciden en que no es nada inofensiva ya que desde lo tecnológico, la innovación transforma en obsoleto aquello que mejora, destruyendo procesos y fábricas de producción ocasionado 
despidos y empobrecimiento, pero a su vez esta obsolescencia constante contribuye por otro lado a aumentar la producción y el empleo ya que nos obliga a cambiar de objetos constantemente y al final la innovación compensa.

Desde la visión cultural el problema crece en la medida que la innovación se anula así misma con su propio éxito, ya que los individuos son la única fuente de valor, con lo que la innovación cesara una vez los individuos queden satisfechos. Es aquí donde los estados y las empresas deben encontrar un equilibrio para estas dos visiones, pero ¿Cómo enfocar las políticas de innovación?, el autor propone una unión de estas dos visiones, donde se fomente la actividad investigativa así como ofrecer las condiciones necesarias para la consolidación de grupos conformados por agentes que promuevan las acciones innovadoras que conlleven al mejoramiento social y económico a partir de la innovación.

\subsection{La Innovación: un factor clave para la competitividad de las empresas}

Confederación Empresarial de Madrid-CEOE Dirección General de Investigación de la Comunidad Autónoma de Madrid

Capítulo 1. El porqué de innovar

Este documento realiza una breve explicación sobre la importancia de la innovación en el crecimiento económico, la competitividad de las empresas y la necesidad de un gobierno que impulse políticas de apoyo, fomento a la innovación, creación de entornos apropiados y la creación de una cultura de la innovación.

\subsubsection{La nueva economía del conocimiento}

Desde hace un par de años se considera que la base fundamental en los países desarrollados son conocimiento y el manejo de la información y son estos factores de crecimiento económico y aumento de la productividad.

Esta nueva economía se define como (CEIM Confederación Empresarial de Madrid-CEOE, 2007) "aquella en la que la generación y explotación del conocimiento juegan un papel predominante en la creación de bienestar". Donde el enorme y rápido desarrollo de las nuevas tecnologías de la información contribuyen a lo que hoy conocemos como: Sociedad de la información.

Esta sociedad se caracteriza por elegir la innovación, el conocimiento y la digitalización como principales motores de crecimiento económico y tecnológico además de la ser las principales fuentes de ventajas competitivas.

Todos estos cambios han generado nuevas formas de empleo y entornos económicos donde la clave para mejorar la calidad de vida se basa en ideas innovadoras aplicadas a nuevos productos (productos, procesos, métodos, etc.) Como se puede observar el término innovación siempre aparece como sinónimo de progreso, bienestar, desarrollo, calidad de vida, por esto que la incorporación 
de una cultura de la innovación se vuelve una necesidad en todos los sectores sociales y económicos de un país.

Aunque es claro que el crecimiento económico y el empleo dependen fundamentalmente de la competitividad empresarial es importante ver la innovación como un estilo de vida social, una forma colectiva de ver las cosas.

\subsubsection{La importancia de la cultura de la innovación}

Un tema tan importante como la misma innovación, es aquel correspondiente a la creación de un entorno propicio que fomento de una verdadera cultura de la innovación en la sociedad.

La trascendencia de este asunto se ve reflejada en las políticas que se gestionan a nivel económico, social, científico y tecnológico, para promover escenarios con fuerte aceptación social hacia la innovación; dejando claro que se debe intensificar los esfuerzos por parte de los gobiernos, en todos su niveles, para facilitar un entorno adecuado donde exista coherencia en las políticas de innovación, la creación de un marco normativo y el fomento de la creación empresarial.

\subsubsection{La dimensión regional de la innovación y el desarrollo tecnológico}

Como se menciona anteriormente, las políticas gubernamentales son esenciales para el desarrollo de una cultura de la innovación, pero es importante referir que estas políticas deben abarcar desde las regiones menos prosperas hasta las empresas, especialmente las Pymes, para brindar el apoyo necesario al fomento de acciones innovadoras.

\subsection{La innovación en el siglo XXI}

Antonio Pulido

Centro de predicción económica Universidad Autónoma de Madrid

La innovación no solo es importante para obtener ganancias de productividad y mejorar la competitividad internacional de las empresas y productos; es también la manera para incrementar el nivel de vida de toda la sociedad y perfeccionar el funcionamiento de todo tipo de instituciones tanto en sus aspectos económicos como extraeconómicos.

Las políticas de estímulo a la innovación deben superar recetas simples, primando la coordinación de acciones público-privadas, los planteamientos globalizadores y las interacciones innovadoras. El complejo y delicado ecosistema de la innovación interrelaciona fuentes, infraestructura, condiciones político-estratégicos y efectos.

Convertir la innovación en un objeto prioritario de la sociedad en su conjunto, exige entenderla en su sentido más amplio que incluye cambios tecnológicos y de organización. Una iniciativa innovadora amplia deberá priorizar el talento, es decir, la creación de conocimiento, la educación y el aprendizaje. Pero también 
difundir esos avances, rentabilizando económica y socialmente los esfuerzos inversores, públicos y privados. (Pulido, 2005)

\subsubsection{Más allá de la innovación}

La innovación, como proceso de creación y transformación del conocimiento, es una nueva solución para los problemas de la humanidad, producto de su propia evolución; a nivel económico se considera como sinónimo de crecimiento y calidad de vida.

Este proceso se caracteriza por añadir a cualquier invento (forma de avanzar en el conocimiento), una capacidad para ser utilizado y así cubrir las necesidades de una comunidad mejorando los niveles de vida.

Dichos niveles van estrechamente relacionados con el aumento de productividad generada por la innovación: tecnológica, de procesos, administrativa y social además de liderar con productos innovadores con alto valor agregado.

Sin embargo, no se debe dejar de lado aspectos como la formación y la calidad del capital humano ya que es garantía de una autentica innovación y de una transformación positiva en la productividad y competitividad. Por esto, es clave que un país considere la innovación como una estrategia para el futuro elevando la eficiencia empresarial, mejorando el entorno de políticas públicas que impulsen la innovación, optimizando los recursos, realizando cambios favorables en la infraestructura e integrando el uso de las TIC.

\subsubsection{Una nueva cultura que debe impregnar a la sociedad en su conjunto}

Si se está convencido de la prioridad económica, social y política de la innovación se debe buscar una estrategia integral para atar todas las fuerzas que deben intervenir en este cambio, dejando claro que la innovación no es solo responsabilidad del gobierno y las empresas; los individuos deben constituir una fuerza promotora y no solo receptores pasivos y en algunos casos opositoras al cambio.

Así mismo la cultura de la innovación, la creación de nuevas ideas y la flexibilidad al cambio constituyen un activo social a cuidar, promover y proteger.

\subsubsection{Una visión global de la innovación}

Ecosistema de la innovación: entorno global compuesto por varios factores que contribuyen a un ambiente innovador. (Pulido, 2005)

\section{Componentes}

- Fuentes de la innovación: está compuesta por los esfuerzos de un país, la cooperación con proveedores y clientes, la adquisición e implantaciones de equipos, la mejora de procesos, colaboración con universidades y especialistas externos, la adquisición de propiedad intelectual.

- Condicionantes político estratégicos: son estímulos a la acción de los diferentes agentes de la innovación que afectan la eficacia general del sistema; incluyes aspectos tales como: un marco estable, la coordinación de 
políticas públicas a diferentes niveles, diseñado integrador de la estrategia publica/privada, entre otras.

- Infraestructura de innovación: sistema que ejecuta potencializa y transmite los esfuerzos financieros de las políticas de apoyo y garantice calidad y cantidad en universidades, redes de formación, instituciones financieras, mano de obra cualificada, etc.

\section{GRÁFICO 1}

Ecosistema de la innovación

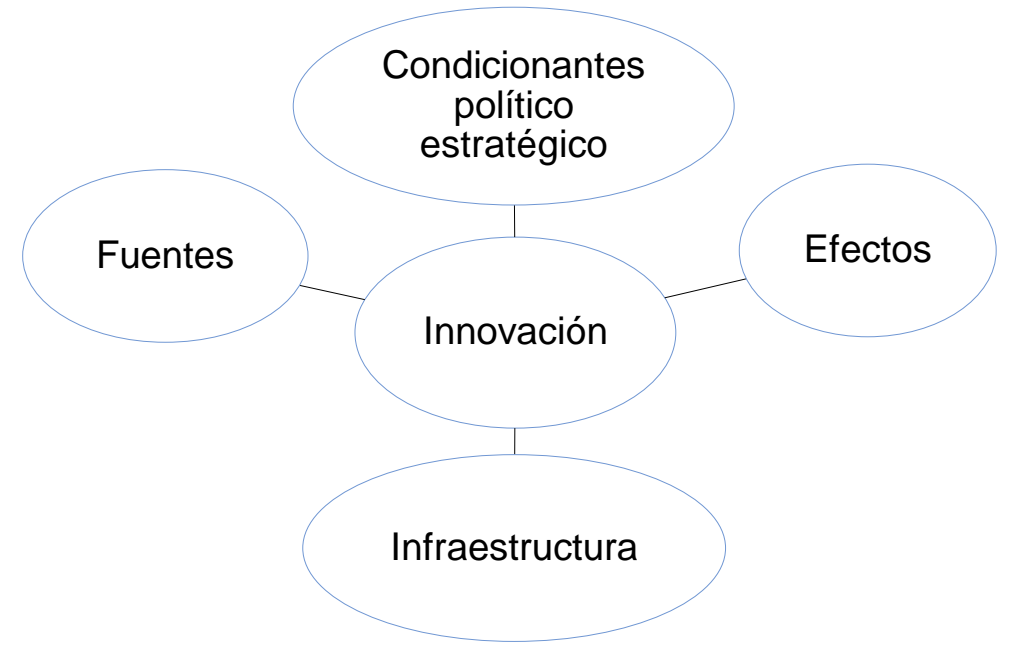

Fuente: (Pulido, 2005)

\subsubsection{Hacia una iniciativa innovadora}

Poner en marcha un proceso de cambio o una iniciativa innovadora implica buscar una acción conjunta en donde se repartan las responsabilidades entre los múltiples agentes con una agenda compartida donde se priorice el talento.

La creación de conocimiento, la educación, el aprendizaje, el mantenimiento de las aptitudes de los individuos, la inversión en investigación y desarrollo son claves para esta iniciativa.

\subsubsection{Los condicionantes políticos - estratégicos}

La innovación requiere de un entorno políticamente favorable para su desarrollo donde existan políticas en diferentes campos como la financiación de I+D+i, incentivos fiscales, apoyo a la transferencia tecnológica y de recursos humano.

\subsection{Emprendimiento e innovación en Colombia: ¿Qué nos está haciendo falta? \\ Profesor Rafael vega Facultad de Administración Universidad de los Andes}

La situación internacional demuestra que los niveles más altos de desarrollo corresponden a países que exhiben altos niveles de emprendimiento e 
innovación en sus economías; presentando un verdadero problema para aquellos países que están lejos de una cultura innovadora, como Colombia, ya que avanzar en una economía tan rápida con entorno tan poco favorable para la innovación hace realmente complejo pertenecer a un entorno competitivo.

Según el Profesor Vega, la innovación y el emprendimiento deben realizar en tres niveles los cuales se muestran en el grafica 2.

Donde afirma que para lograr una sociedad más emprendedora es necesario activar el potencial de cada nivel y conseguir pasar de las consideraciones analíticas a verdaderas estrategias que involucren los tres campos por medio de conexiones efectivas para incrementar la fuerza de la innovación emprendedora en el país.

\section{GRÁFICO 2}

Niveles de análisis

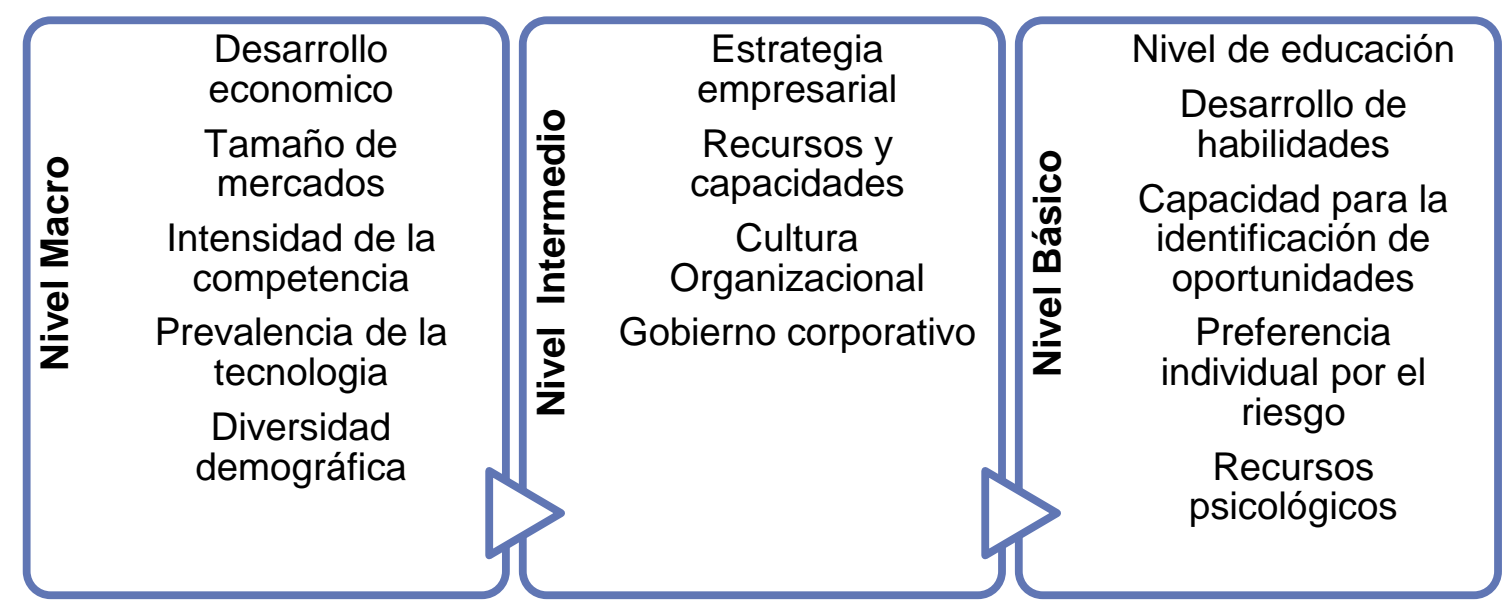

Fuente: (Vesga, 2006)

Estas variables fueron estudiadas en Colombia para establecer los puntos críticos de nuestro país, planteando el avance obligado en temas como infraestructura e innovación, la construcción de redes de emprendedores, organizar esfuerzos para desarrollar una cultura emprendedora. Todo esto arrojado desde el análisis al Informe de competitividad y otros estudios como el realizado por el centro de productividad del pacifico.

\section{MÉTODO}

Para cumplir con los propósitos de este trabajo se llevara a cabo la lectura de algunos documentos que orienten y aporten al desarrollo del tema; además se apoyará la investigación con un estudio de caso exploratorio donde se implementó una entrevista informal, la cual se encuentra en el anexo 1, al profesor Gabriel Izard y se planteó unas preguntas para la visita empresarial a Barcelona activa, las cuales fueron resultas en la conferencia con Marc Sans Guañabens asesor de promoción de Barcelona activa. 


\section{RESULTADOS}

\subsection{Universidad Autónoma De Barcelona}

Alisson Núñez: ¿Por qué escoger la innovación como pilar para el crecimiento? Gabriel Izard: Para ser diferentes, si tú no eres diferente no puedes crecer, sí no innovas no puedes ser diferente.

Alisson Núñez: Cómo es conocido Barcelona se ha destacado en últimos años por ser una de las ciudades innovadora, ¿Cuál cree que han sido las acciones que llevaron a dicho reconocimiento?

Gabriel Izard: Mucho esfuerzo por parte de las empresas y de las administraciones es público para conseguir atraer talento, cuidarlo y realizar todas las actividades como las de Barcelona activa, es un tema de esfuerzo.

Alisson Núñez: ¿Cómo lograr que la sociedad se comprometan con la innovación y se prepare para el cambio?

Gabriel Izard: Es una cosa complica, pero para mí la parte más importante es lo que se hace poco, la formación. La formación es lo más importante para que la innovación se vaya generando y se vaya reproduciendo, si no hay formación no hay gente con talento. Formación sobre cualquier tema más inversión en escuelas, universidades todo lo que está detrás del conocimiento; no hay innovación si no hay conocimiento.

\subsection{BARCELONA ACTIVA}

¿Cuál fue el objetivo inicial para crear esta organización? ¿Sigue siendo el mismo?

Marc Sans: Barcelona activa nace en diciembre de 1886 siendo la primera incubadora de empresas con el objetivo de impulsar el crecimiento económico de Barcelona apoyando a las empresas, brindando formación en varias áreas, brindando empleo y atrayendo talento internacional.

¿Considera que tanto en la organización como en Barcelona existe una cultura innovadora?

Marc Sans: Si contamos con muchas empresas innovadoras, en este momento tenemos 52 empresas utilizando nuestro servicio de incubación hemos recibido alrededor de 11.100 en las sesiones de bienvenida pero considero que podemos explotar más sectores estratégicos.

¿Cuáles son los factores clave para fomentar la innovación?

Marc Sans: Contar con el apoyo, la iniciativa y el espacio adecuado para llevar a cabo nuestras ideas.

¿Cómo identificar y explorar las mejores y más fértiles oportunidades de innovación?

Marc Sans: Aquí en Barcelona activa medimos el grado de innovación de cada proyecto con un grupo de experto que la mide de acuerdo al estado del arte. Por ejemplos si son parámetros tecnológicos nos apoyamos de los expertos con los que tenemos convenio. Nosotros observamos el plan de negocios y su viabilidad; también nos fijamos en el curriculum y la integración del grupo. 
¿Cuáles son los aportes sociales y económicos que ofrece la empresa?

Marc Sans: A nivel económico hemos ayudado a la creación de 1.900 empresas creando 3.400 puestos de trabajo, ayudando a gran cantidad de personas a encontrar empleo gracias nuestro servicio de búsqueda de empleados además de contribuir al desarrollo de la ciudad con nuestras estrategias promoción; a nivel social considero importante la ayuda prioritaria con los Programas para sectores con necesidades especiales.

\section{CONCLUSIONES}

De acuerdo con el conocimiento adquirido en el seminario y las visitas realizadas se puede observar el interés generalizado por la innovación y la iniciativa por aportar al crecimiento de la ciudad por medio de la generación de ideas innovadoras que contribuyan a la creación de empresa.

Las respuestas obtenidas contribuyen al objetivo de este trabajo, afirmando que la innovación debe ser escogida como pilar para el crecimiento y debe ser promovida a través de la formación, ya que es un área clave para logar comprometer a la sociedad, impulsando la inversión en universidades, escuelas y otros centros de estudio.

Así como lo menciona el profesor Izard, existen varias organizaciones que realizan actividades para apoyar iniciativas emprendedoras y una de esas es Barcelona Activa, la cual tiene como propósito ayudar a todas aquellas personas con ideas creativas con el fin de para posicionar a Barcelona como referente de ciudad emprendedora, apoyando a las empresas para mejorar su competitividad y fomentar la generación de riqueza y empleo.

Esta agencia de desarrollo social basa sus áreas de actividad ${ }^{1}$ de acuerdo los siguientes pilares:

- Creación de empresas y desarrollo de una cultura empresarial: el objetivo es proporcionar servicios profesionales para emprendedores potenciales con el fin de fomentar la creación de nuevos negocios, así como buscar incentivar el desarrollo de una cultura empresarial.

- Consolidación y crecimiento de empresas innovadoras: busca generar las condiciones que potencien el crecimiento de empresas innovadoras en la ciudad a través de la internacionalización, acceso al financiamiento, cooperación e innovación.

- Desarrollo de capital humano y nuevas oportunidades de empleo: el objetivo es promover a Barcelona Activa como punto de encuentro para el capital humano en la ciudad, con el fin de aumentar las oportunidades

\footnotetext{
${ }^{1}$ Empresa, Emprendimiento, Capacitación profesional y empleo, Formación, Promoción de ciudad, Promoción de los sectores estratégicos, Promoción del turismo y eventos, Promoción del territorio y del comercio, Recursos Económicos, Recursos Humanos, Sistemas de Información y Organización, Servicios Jurídicos y Comunicación y Marketing.
} 
profesionales que la nueva cultura de trabajo, las nuevas ocupaciones y los mercados económicos emergentes pueden ofrecer a la población.

- Acceso y mejoras de empleo: Barcelona Activa busca informar, orientar, motivar, capacitar y promover el empleo en Barcelona.

- Comunicación y Marketing: participar en la definición y ejecución de la política de comunicación y de marketing de Barcelona Activa.

Donde en el informe "Memoria de actividades 2013" (Ayuntamiento de Barcelona. Barcelona Activa, 2014, pág. 3), se recopila los resultados de todas las actividades llevadas a cabo por Barcelona activa.

En el terreno económico, la ciudad registró una dinámica positiva en creación de nuevas empresas con la creación de más de 7.000 sociedades mercantiles, un $5 \%$ más que el año anterior y un sustancial aumento del volumen de inversión extranjera.

En el ámbito de la internacionalización, el comportamiento del turismo en el 2013 fue muy positivo dejando nuevos máximos históricos de pernoctaciones y turistas alojados en establecimientos hoteleros, mientras que el gasto de compra internacional con tarjeta de crédito y el número de pasajeros en cruceros han evolucionado con dinamismo (con variaciones interanuales del $+28,7 \%$ y $+7,9 \%$, respectivamente).

Asimismo, con recursos propios, el Ayuntamiento de Barcelona ha garantizado, a través de Barcelona Activa, un conjunto de servicios permanentes y universales a las personas que buscan trabajo y a las empresas, de promoción del territorio y de la marca Barcelona en clave económica, unos servicios eficaces, bien dimensionados, de calidad y con adaptabilidad para hacer frente a las actuales necesidades, pero también para avanzar hacia un modelo económico competitivo, sostenible y con calidad de vida para las personas.

Este panorama nos demuestran la obligación de desarrollar estrategias para incorporar el concepto de innovación en la sociedad, donde cada persona entienda y vea la necesidad de empezar a generar ideas creativas las cuales se puedan llevar a cabo gracias a un gobierno que establezca políticas que faciliten su desarrollo y puesta en marcha.

Como lo afirma el empresario Manuel José Moreno ( Díaz Sandoval, 2013) "el éxito en las empresas no solo depende de administrar con eficiencia los recursos sino de cumplir con una tarea inaplazable: innovar.".

Y es aquí donde debemos analizar nuestra situación para medir y estudiar que nos hace falta.

Según el informe de competitividad global (Schwab, 2014), estudio que se encarga de medir la competitividad de 144 países por medio del análisis de 12 pilares para presentar las principales fortalezas y debilidades de cada una de las economías, Colombia ocupa el puesto 66 en el listado general, 78 en el 
subíndice: requerimientos básicos ${ }^{2}, 63$ en el subíndice: factores que mejoran la eficiencia ${ }^{3}$ y 64 en el subíndice: Factores de innovación y sofisticación. ${ }^{4}$

Aunque el pilar de innovación no es el peor en nuestro país, si ocupa un lugar muy bajo, el 77 en el informe y se encuentra en los seis peores calificados.

Esta calificación se debe a la poca capacidad para innovar de las empresas, la baja inversión en investigación y desarrollo y las escasas solicitudes presentadas en materia de Patentes.

Inicialmente nuestro país debe iniciar con la diversificación en la economía y ser menos dependientes a los ingresos obtenidos gracias a los recursos naturales, y como se comenta en el informe, debemos construir un ecosistema de innovación más sólido que requerirá no sólo más y mejor inversión pública, sino también un reconocimiento decisivo por parte de las empresas colombianas de la necesidad de innovar mediante la realización de un conjunto de inversiones en áreas tales como la I + D, así como programas de formación en el puesto de trabajo y la adopción de las TIC.

Según Alessandro Maffioli, experto internacional del BID, (COLCIENCIAS , 2015) Un estudio realizado por el Banco Interamericano de Desarrollo al Programa de Innovación de Colciencias, concluyó que los proyectos financiados entre 1995 y 2007 tuvieron un impacto del $15 \%$ en la productividad empresarial y otro del $12 \%$ en el número de productos.

Demostrando que los esfuerzos realizados por el país en el diseño, la articulación y estimulación de políticas han dado resultados pero no lo suficientes para el lograr consolidar la innovación en la sociedad.

Por esto se propone una serie de medias, de acuerdo a la información recopilada en esta ponencia, que generen impactos positivos sobre la actividad económica del país y ayuden al fomento de la cultura de innovación en Colombia.

- Establecer a largo plazo estrategias que aporten a la sofisticación y diversificación de la economía alineando los objetivos de los diferentes agentes público y privado para logar implantar estrategias basadas en "Smart specialization".

- Definir un sistema gubernamental solido que se encargue del control de los recursos y a si mismo del cumplimiento de metas con un elevado grado

\footnotetext{
2 Este grupo está conformado por los pilares: Instituciones, Infraestructura, Entorno macroeconómico y Salud y educación primaria.

${ }^{3}$ Este grupo está conformado por los pilares: Educación superior y capacitación, Eficiencia del mercado de bienes, Eficiencia del mercado laboral, Desarrollo del mercado financiero, Preparación tecnológica y Tamaño del mercado.

${ }^{4}$ Este grupo está conformado por los pilares: Sofisticación de los negocios e Innovación.

${ }^{5}$ Políticas de crecimiento basado en lugar-región y su objetivo es mejorar la asignación de la inversión pública en I $+\mathrm{D}$ e inversiones relacionadas con la innovación, con el fin de estimular la competitividad, la productividad y el crecimiento económico a través de las actividades empresariales. (OCDE, 2012).
} 
de transparencia en la gestión y evaluación continua de los planes proyectos y estrategias.

- Promover estrategias que fomenten la formación gerencial de aquellas empresas poco innovadoras para incrementar la productividad del país.

- Invertir en el fortalecimiento de la cultura innovadora por medio de actuaciones de carácter académico, científico, tecnológico, artístico y cultural, así como fomentar el intercambio de conocimiento e información.

- Construir programas flexibles que cumplan con las necesidades de diferentes usuarios de acuerdo a un perfil específico.

- Fomentar espacios especializados en emprendimiento e innovación, en los cuales se brinden sesiones formativas, asesoría y capacitación. en temas relacionado con constitución de empresas, información y trámites, financiación empresarial, búsqueda de trabajadores, internacionalización y trámites de comercio exterior e innovación.

Para cambiar hay que tener la motivación, todos los países necesitan crecimiento económico y desarrollo social teniendo en cuenta que en la actualidad la innovación es la nueva forma de medir la riqueza en los países. 


\section{BIBLIOGRAFÍA}

Díaz Sandoval , M. (02 de Agosto de 2013). Sólo el 9\% de las empresas en Colombia son innovadoras. El Espectador, pág. 1.

Alcina Franch, J. (1989). Arqueología antropológica. Madrid: Akal S.A.

Ayuntamiento de Barcelona. Barcelona Activa. (2014). Memoria de Actividades 2013. Barcelona.

Barcelona activa. (2014). Barcelona Data Sheet 2013. Barcelona: Area de economía, empresa y ocupación.

Barcelona Activa. (s.f.). Barcelona Activa. Recuperado el 27 de Mayo de 2015 , de http://www.barcelonactiva.cat/barcelonactiva/es/index.jsp

BBVA. (19 de Semptiembre de 2012). BBVA Con tu empresa. Recuperado el 11 de Julio de 2015, de http://www.bbvacontuempresa.es/a/la-teoria-del-empresario-innovadorschumpeter

CEIM Confederación Empresarial de Madrid-CEOE. (20 de Julio de 2007). La Innovación:un factor clave para la competitividad de las empresas. 9. (D. G. Madrid, Ed.) Madrid.

COLCIENCIAS . (14 de Julio de 2015). COLCIENCIAS: ciencia, tecnología e innovación. Obtenido de http://www.colciencias.gov.co/noticias/la-innovaci-n-en-colombia-seleva-la-productividad-empresarial

Consejo privado de Competitividad . (2014). Informe nacional de competitividad 2014-2015: Ciencia, Tecnología e Innovación. Bogotá: Consejo privado de Competitividad .

Departamento Nacional de Planeación. (2015). Reporte Global de Competitividad del Foro Económico Mundial 2014-2015: Resultados para Colombia. Bogotá.

Izard, G. (06 de Junio de 2015). La cultura de la innovación en Barcelona. (A. A. Núñez Martínez, Entrevistador)

McCurdy, D., \& Spradley, J. P. (1975). Anthropology: The Cultural Perspective. New York: John Wiley and Sons.

Menéndez Viso, A. (Febrero de 2013). Innovación y cultura: la medida social de la innovación. ISEGORIA.Revista de Filosofía Moral y Política(48), 185-196.

OCDE. (1997). Manual de Oslo: Guia para la recolección e interpretación de datos sobre la innovacion.

OCDE. (2012). Draft synthesis report on innovation driven-growth in regions:the role of smart specialisation. OCDE.

Pulido, A. (2005). La Innovació en el Siglo XXI. (C. d. (CEPREDE), Ed.) Madrid. 
Real Academia Española. (09 de Julio de 2015). Real Academia Española. Obtenido de http://lema.rae.es/drae/?val=innovacion

Rodriguez Rios, L. (10 de Mayo de 2010). Premio Nacional de Tecnología e Innovación . Recuperado el 28 de Mayo de 2015, de http://www.fpnt.org.mx/boletin/Mayo_2010/Pdf/Cultura_de_Innovacion.pdf

Schwab, K. (2014). The global competitiveness report 2014-2015. Geneva: World Economic Forum.

Soto Flores, M. d., \& Medellín Cabrera, E. A. (2010). La innovación y el empresario innovador en Drucker. Congreso Internacional de Sistemas de Innovación para la Competitividad 2010, (págs. 1-19). Guanajuato de Salvatierra.

Veiga, L. (2008). Innovación y competitividad. Revista de Antiguos Alumnos del IEEM , $72-$ 87.

Vesga, R. (06 de Junio de 2006). Centro de Estrategia y Competitividad CEC. Recuperado el 27 de Mayo de 2015, de http://cec.uniandes.edu.co/index.php/articulos 


\section{Anexo 1}

\section{ENTREVISTA INFORMAL}

Propósito: Conocer la opinión de los expertos sobre la innovación como factor de desarrollo empresarial y herramienta para el crecimiento de la ciudad. Además de explorar otros factores importantes que influyen para promover una cultura innovadora.

Las siguientes preguntas serán utilizadas para tener un enfoque en la charla pero se espera obtener más información de acuerdo a las respuestas. Proporcionadas por el entrevistado.

Dirigido a:

- $\quad$ Docente (es) Universidad Autónoma De Barcelona

- Barcelona Activa

Preguntas guía:

\section{DOCENTES}

- ¿Por qué escoger la innovación como pilar para el crecimiento?

- Como es conocido Barcelona se ha destacado en últimos años por ser una de las ciudades innovadora, ¿Cuál cree que han sido las acciones que llevaron a dicho reconocimiento?

- ¿Cómo lograr que la sociedad se comprometan con la innovación?

- ¿Cómo preparar al capital humano para el cambio?

\section{BARCELONA ACTIVA}

- ¿Cuál fue el objetivo inicial para crear esta organización? ¿Sigue siendo el mismo?

- ¿Considera que tanto en la organización como en Barcelona existe una cultura innovadora?

- ¿Cuáles son los factores más importantes a la hora de Innovar?

- ¿Cómo identificar y explorar las mejores y más fértiles oportunidades de innovación

- ¿Cuáles son los aportes sociales y económicos que ofrece la empresa? 